\title{
Syndrome Development to Assess IDU, HIV, and Homelessness in MA Emergency Departments
}

\section{Stefanie P. Albert, Rosa Ergas, Sita Smith, Gillian Haney, Monina Klevens}

Bureau of Infectious Disease and Laboratory Sciences, Massachusetts Department of Public Health, Boston, Massachusetts, United States

Objective

We sought to measure the burden of emergency department (ED) visits associated with injection drug use (IDU), HIV infection, and homelessness; and the intersection of homelessness with IDU and HIV infection in Massachusetts via syndromic surveillance data.

\section{Introduction}

In Massachusetts, syndromic surveillance (SyS) data have been used to monitor injection drug use and acute opioid overdoses within EDs. Currently, Massachusetts Department of Public Health (MDPH) SyS captures over 90\% of ED visits statewide. These real-time data contain rich free-text and coded clinical and demographic information used to categorize visits for population level public health surveillance.

Other surveillance data have shown elevated rates of opioid overdose related ED visits, Emergency Medical Service incidents, and fatalities in Massachusetts from 2014-2017 [1-3]. Injection of illicitly consumed opioids is associated with an increased risk of infectious diseases, including HIV infection. An investigation of an HIV outbreak among persons reporting IDU identified homelessness as a social determinant for increased risk for HIV infection.

\section{Methods}

To accomplish our objectives staff used an existing MDPH SyS IDU syndrome definition [4], developed a novel syndrome definition for HIV-related visits, and adapted Maricopa County's homelessness syndrome definition. Syndromes were applied to Massachusetts ED data through the CDC's BioSense Platform.

Visits meeting the HIV and homelessness syndromes were randomly selected and reviewed to assess accuracy; inclusion and exclusion criteria were then revised to increase specificity. The final versions of all three syndrome definitions incorporate freetext elements from the chief complaint and triage notes, as well as International Statistical Classification of Diseases and Related Health Problems, 9th (ICD-9) and 10th Revision (ICD-10) diagnostic codes. Syndrome categories were not mutually exclusive, and all reported visits occurring at Massachusetts EDs were included in the analysis.

\section{Syndromes Created}

For the HIV infection syndrome definition, we incorporated the free-text term "HIV" in both the chief complaint and triage notes. Visit level review demonstrated that the following exclusions were needed to reduce misspellings, inclusion of partial words, and documentation of HIV testing results: "negative for HIV", "HIV neg", "negative test for HIV", "hive", "hivies", and "vehivcle". Additionally, the following diagnostic codes were incorporated: V65.44 (Human immunodeficiency virus [HIV] counseling), V08 (asymptomatic HIV infection status), V01.79 (contact with or exposure to other viral diseases), 795.71 (nonspecific serologic evidence of HIV), V73.89 (special screening examination for other specified viral diseases), 079.53 (HIV, type 2 [HIV-2]), Z20.6 (contact with and (suspected) exposure to HIV), Z71.7 (HIV counseling), B20 (HIV disease), Z21 (asymptomatic HIV infection status), R75 (inconclusive laboratory evidence of HIV), Z11.4 (encounter for screening for HIV), and B97.35 (HIV-2 as the cause of diseases classified elsewhere).

Building on the Maricopa County homeless syndrome definition, we incorporated a variety of free-text inclusion and exclusion terms. To meet this definition visits had to mention: "homeless", or "no housing", or, "lack of housing", or "without housing", or "shelter" but not animal and domestic violence shelters. We also selected the following ICD-10 codes for homelessness and inadequate housing respectively, Z59.0 and Z59.1.

We analyzed MDPH SyS data for visits occurring from January 1, 2016 through June 30, 2018. Rates per 10,000 ED visits categorized as IDU, HIV, or homeless were calculated. Subsequently, visits categorized as IDU, HIV, and meeting both IDU and HIV syndrome definitions (IDU+HIV) were stratified by homelessness. 


\section{Results}

\section{Syndrome Burden on ED}

The MDPH SyS dataset contains 6,767,137 ED visits occurring during the study period. Of these, 82,819 (1.2\%) were IDU-related, $13,017(0.2 \%)$ were HIV - related, $580(<0.01 \%)$ were related to IDU + HIV, and 42,255 visits $(0.6 \%)$ were associated with homelessness.

The annual rate of IDU-related visits increased 15\% from 2016 through June of 2018 (from 113.63 to 130.57 per 10,000 visits); while rates of HIV-related and IDU

+ HIV-related visits remained relatively stable. The overall rate of visits associated with homelessness increased $47 \%$ (from 49.99 to 73.26 per 10,000 visits). Rates of IDU, HIV, and IDU + HIV were significantly higher among visits associated with homelessness. Among visits that met the homeless syndrome definition compared to those that did not: the rate of IDU-related visits was 816.0 versus 118.03 per $10,000 \mathrm{ED}$ visits $(\mathrm{X}[2]=547.12, \mathrm{p}<0.0001)$; the rate of visits matching the HIV syndrome definition was 145.54 versus 18.44 per $10,000 \mathrm{ED}$ visits $(\mathrm{X}[2]=99.33$, $\mathrm{p}<0.0001)$; and the rate of visits meeting the IDU+HIV syndrome definition was 15.86 versus 0.76 per 10,000 visits $(X[2]=13.72, p=0.0002)$.

\section{Conclusions}

Massachusetts is experiencing an increasing burden of ED visits associated with both IDU and homelessness that parallels increases in opioid overdoses. Higher rates of both IDU and HIV-related visits were associated with homelessness. An understanding of the intersection between opioid overdoses, IDU, HIV, and homelessness can inform expanded prevention efforts, introduction of alternatives to ED care, and increase consideration of housing status during ED care.

Continued surveillance for these syndromes, including collection and analysis of demographic and clinical characteristics, and geographic variations, is warranted. These data can be useful to providers and public health authorities for planning healthcare services.

\section{Acknowledgement}

Special thanks to the Maricopa County Department of Public Health, and the following Massachusetts Department of Public Health Bureau of Infectious Disease and Laboratory Sciences staff: Mark Bova, Katherine Brown, Dan Church, Kevin Cranston, Alfred DeMaria, and Shauna Onofrey.

\section{References}

1. Vivolo-Kantor AM, Seth P, Gladden RM, et al. 2018. Vital Signs: Trends in Emergency Department Visits for Suspected Opioid Overdoses — United States, July 2016-September 2017. MMWR Morb Mortal Wkly Rep. 67(9), 279-85. doi:https://doi.org/10.15585/mmwr.mm6709e1. PubMed

2. Massachusetts Department of Public Health. Chapter 55 Data Brief: An assessment of opioid-related deaths in Massachusetts, 2011-15. 2017 August. Available from: https://www.mass.gov/files/documents/2017/08/31/data-briefchapter-55-aug-2017.pdf

3. Massachusetts Department of Public Health. MA Opioid-Related EMS Incidents 2013-September 2017. 2018 Feb. Available from: https://www.mass.gov/files/documents/2018/02/14/emergency-medical-services-data-february-2018.pdf

4. Bova M. Using emergency department (ED) syndromic surveillance to measure injection-drug use as an indicator for hepatitis C risk. Powerpoint presented at: 2017 Northeast Epidemiology Conference. 2017 Oct 18 - 20; Northampton, Massachusetts, USA. 
ISDS 2019 Conference Abstracts

Rate of IDU, HIV, and IDU+HIV Related Visits Captured by MDPH SyS per 10,000 ED Visits by Year (2016 through 2018*)

\begin{tabular}{|c|c|c|c|c|c|}
\hline Year & $\begin{array}{c}\text { IDU-Related } \\
\text { Visits }\end{array}$ & $\begin{array}{c}\text { HIV-Related } \\
\text { Visits }\end{array}$ & $\begin{array}{r}\text { Meets IDU and HIV } \\
\text { Definitions } \\
(\text { IDU+HIV) }\end{array}$ & Homeless Visits & $\begin{array}{l}\text { All MDPH SyS } \\
\text { ED Visits (N) }\end{array}$ \\
\hline 2016 & 113.63 & 16.89 & 0.59 & 49.99 & $2,367,004$ \\
\hline 2017 & 125.19 & 20.56 & 0.95 & 66.88 & $2,842,048$ \\
\hline $\begin{array}{c}2018 \\
\text { Jan- June* }\end{array}$ & 130.57 & 20.39 & 1.08 & 73.26 & $1,558,085$ \\
\hline TOTAL & 122.38 & 19.24 & 0.86 & 62.44 & $6,767,137$ \\
\hline
\end{tabular}

* Data are current as of $8 / 19 / 2018$ and may be subject to change. 